\title{
Cooperative Behavior and Pattern Formation in Mixtures of Driven and Non-Driven Colloidal Assemblies
}

\author{
C. Reichhardt and C.J. Olson Reichhardt \\ Center for Nonlinear Studies and Theoretical Division, \\ Los Alamos National Laboratory, Los Alamos, New Mexico 87545
}

(Dated: September 19, 2018)

\begin{abstract}
We simulate a disordered assembly of particles interacting through a repulsive Yukawa potential with a small fraction of the particles coupled to an external drive. Distortions in the arrangement of the nondriven particles produce a dynamically induced effective attraction between the driven particles, giving rise to intermittent one-dimensional stringlike structures. The velocity of a moving string increases with the number of driven particles in the string. We identify the average stable string length as a function of driving force, background particle density, and particle charge. This model represents a new type of collective transport system composed of interacting particles moving through deformable disorder.

PACS numbers: 82.70.Dd
\end{abstract}

The collective transport of interacting particles driven through disordered backgrounds has been studied extensively in systems such as moving vortex lattices in the presence of random pinning 1], driven charge density waves [2], and sliding friction [3]. A variety of nonequilibrium behaviors occur in these systems, including fractal flow patterns, avalanches, nonlinear velocity-force responses, and dynamic reordering transitions. The collective effects arise due to the particle-particle interactions, while the disorder in the substrate is quenched and does not change with time. It is also possible to have substrates which can distort and respond to the motion of the driven particles, such as in the case of a single colloidal particle driven through a disordered background of other particles which are not coupled to the external drive [4, 5]. Both simulations [5] and experiments [4] show that such a system can exhibit a nonzero threshold force for motion as well as a nonlinear velocity vs applied force response. The driven particle moves in an intermittent manner and causes local rearrangements and distortions in the surrounding bath of colloids. An open question is what happens if there are multiple particles driven through a background of nondriven particles, rather than only a single driven particle. In particular, it is possible that the ability of the surrounding media to react to the motion of driven particles could induce an emergent effective driven particle-particle interaction, leading to new types of moving structures or to pattern formation that is not observed in driven systems with fixed or quenched background disorder.

Recent studies have focused on a related system in which two sets of identical repulsive particles are driven in opposite directions [ 6, , $, 8,9]$. Here, a laning phenomenon occurs, and the species segregate into multiple streams flowing past one another. The oppositely driven species model has been studied both in terms of pedestrian dynamics, where the particles represent people moving in opposite directions [8], as well as in the context of colloidal or charged Yukawa particles in the presence of a driving field [7]. The colloidal systems are particularly attractive for further study since binary mixtures of oppositely charged colloids which can undergo laning transitions in the presence of a driving field have been produced recently in experiment [9]. This type of system opens a wealth of new experimental possibilities.

To our knowledge, the case of a small fraction of particles driven through a responsive but undriven background has not been studied previously. In this work, we show that when multiple particles move through a background of nondriven particles, an effective emergent attraction arises between the driven particles despite the fact that all of the pairwise interactions between the particles are repulsive. We find that intermittent moving strings form which have an average stable length that is a function of the driving force, system density, and particle charge. Strings that are longer than average are very short lived. The string velocity increases monotonically with the string length.

We consider a two-dimensional system with periodic boundary conditions in the $x$ and $y$ directions containing $N_{c}$ particles interacting via a screened Coulomb or Yukawa interaction. Only a small number $N_{D}$ of the particles couple to an external drive. The particles move in an overdamped background, and we neglect hydrodynamic effects, which is reasonable for the low volume fraction limit considered here. We use molecular dynamics (MD) to numerically integrate the overdamped equations of motion for the particles, given for particle $i$ by $d \mathbf{r}_{i} / d t=$ $\mathbf{F}_{i}^{c c}+\mathbf{F}_{i}^{D}+\mathbf{F}_{i}^{T}$. The colloid-colloid interaction force term $\mathbf{F}_{i}^{c c}=-q_{i} \sum_{j \neq i}^{N_{c}} \nabla_{i} V\left(r_{i j}\right)$ contains the Yukawa interaction potential $V\left(r_{i j}\right)=\left(q_{j} /\left|\mathbf{r}_{i}-\mathbf{r}_{j}\right|\right) \exp \left(-\kappa\left|\mathbf{r}_{i}-\mathbf{r}_{j}\right|\right)$, where $\mathbf{r}_{i(j)}$ is the position of particle $i(j), q_{i(j)}$ is the charge of colloid $i(j)$, and all colloids have the same sign of charge so that all interaction forces are repulsive. The screening length $1 / \kappa$ is set to $1 / 2$ in all our simulations. The background colloids are composed of a 50:50 mix- 


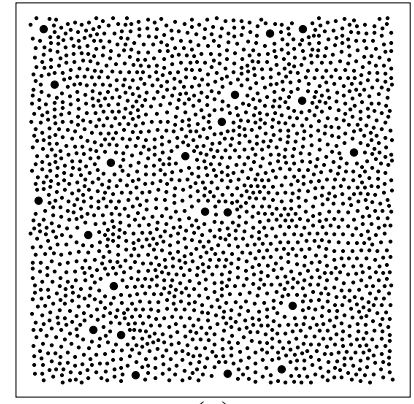

(a)

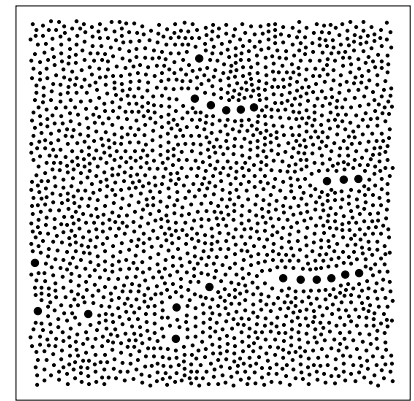

(c)

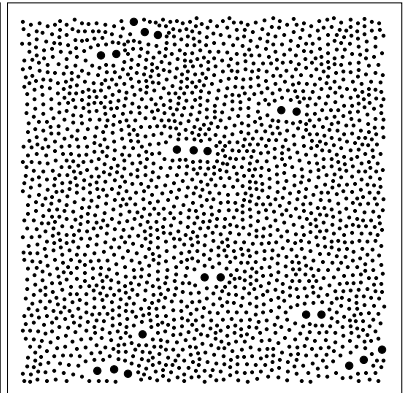

(b)

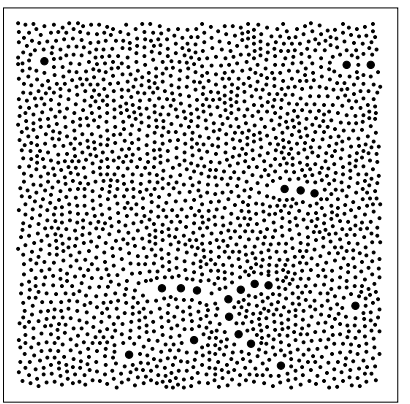

(d)
FIG. 1: Images of the system composed of a disordered array of background colloids (small circles) and particles that couple to the external drive (large circles) for $q_{D} / q=6.67, n_{c}=0.94$, $F_{D}=3.0$, and $N_{D} / N_{c}=0.01$. (a) Initial configuration; (b) the same system after $10^{5} \mathrm{MD}$ steps; (c) a typical steady state snapshot after $10^{6}$ MD steps; (d) snapshot of a string breaking into shorter strings. The driving force is from left to right.

ture of two charges with $q_{1} / q_{2}=1 / 2$, which produces a noncrystalline background arrangement. The average background charge $q=\left(q_{1}+q_{2}\right) / 2$. For the non-driven particles, the constant drive force term $\mathbf{F}_{D}^{i}=0$. The colloids that couple to the external field have charge $q_{D}$ and experience a constant drive $\mathbf{F}_{D}=F_{D} \hat{\mathbf{x}}$ applied after the system is equilibrated by simulated annealing. The thermal force $\mathbf{F}_{i}^{T}$ has the properties $\left\langle\mathbf{F}_{i}^{T}\right\rangle=0$ and $\left\langle\mathbf{F}_{i}^{T}(t) \mathbf{F}_{j}^{T}\left(t^{\prime}\right)\right\rangle=2 \pi \eta k_{B} T \delta_{i j} \delta\left(t-t^{\prime}\right)$. For a system of length $L$ the colloid density is $n_{c}=N_{c} / L$. We consider both the constant $n_{c}$ case, where $L$ and $N_{c}$ are increased simultaneously, as well as the case where $n_{c}$ is increased by fixing $L$ and increasing $N_{c}$.

In Fig. 1 we show four snapshots which highlight the dynamically induced attraction of the driven particles, indicated as large black dots, and the formation of the string structures. Here the density $n_{c}=0.94$, $q_{D} / q=6.67$, and $F_{D}=3.0$. Fig. 1 (a) shows the initial state in which the driven particles are well separated from each other. In Fig. 1(b), after $10^{5}$ MD time steps, the driven particles begin to form string like structures of length $N_{s}=2$ to 3 colloids which are aligned in the direction of the driving force (left to right in the figure). In this nonequilibrium system, despite the fact that all of the pair-wise interactions are repulsive, an effective attraction emerges between the driven particles. What is difficult to convey through still images is the fact that
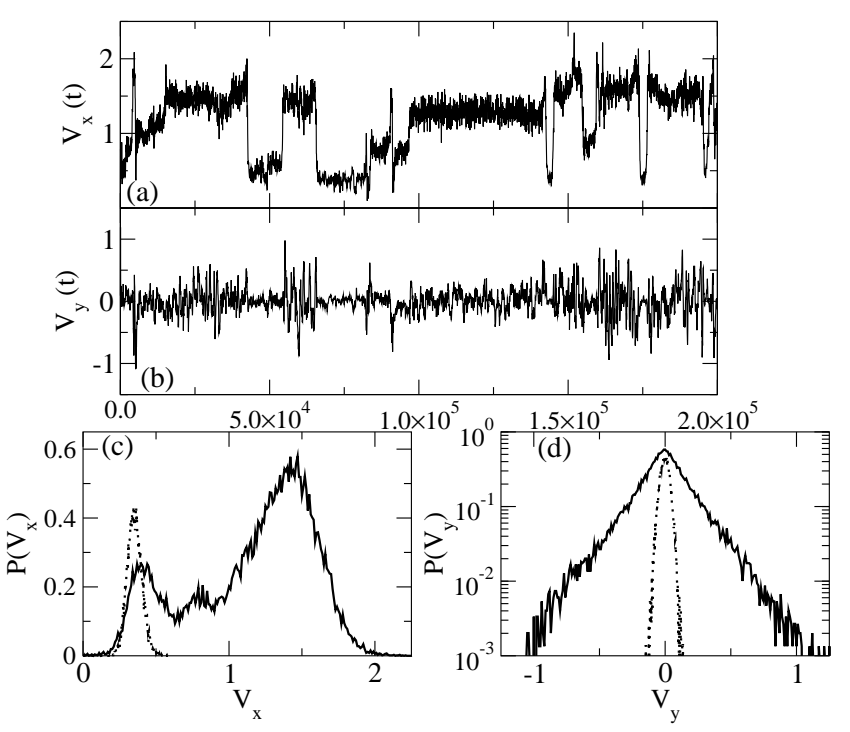

FIG. 2: (a) Time series $V_{x}(t)$ for a single driven colloid from the system in Fig. 1(a) with $N_{D}=20$. (b) The corresponding time series for $V_{y}$. (c) Solid line: The normalized histogram of $V_{x}$ from the complete time series for the system in (a). Dotted line: Histogram of $V_{x}$ for the case $N_{D}=1$. (d) Solid line: The normalized histogram of $V_{y}$ for the total time series from the system in (b). Dotted line: Histogram of $V_{y}$ for the case $N_{D}=1$.

as $N_{s}$, the number of particles in a string, increases, the average string velocity $\left\langle V_{x}^{s}\right\rangle$ increases so that strings move considerably faster than isolated driven particles. In Fig. 1(c) after $10^{6} \mathrm{MD}$ time steps, the system has reached a nonequilibrium steady state. Here the average stable length of a string $\left\langle N_{s}\right\rangle=5$ to 6 . Strings with $N_{s}>6$ form occasionally but generally have a very short lifetime. In Fig. 1(c), strings with $N_{s}=6,5,3$, and 1 are present. All of the strings have an intermittent character, in that driven particles in the string can become detached from the string, and new driven particles can join the string. Typically strings add or shed one driven particle at a time; however, long strings with $N_{s}=7$ or 8 tend to break up into separate strings with $N_{s}=3,4$, or 5, as illustrated in Fig. 1(d).

We note that if $N_{D} / N_{c}$ is increased while all other parameters are held fixed, then $\left\langle N_{s}\right\rangle$ remains unchanged at $\left\langle N_{s}\right\rangle=5$ to 6 . We find the formation of stable strings over an extensive range of parameters, including all $q_{D} / q \geq 1.0$ (where we have checked up to $q_{D} / q=40$ ), for $n_{c}>0.17$, and for arbitrarily large $F_{D}$. The value of $\left\langle N_{s}\right\rangle$ depends on the specific choice of parameters; however, the phenomenon of string formation is very robust provided that the driven particles can distort the arrangement of the background particles. In Fig. 1, $N_{D} / N \approx 0.01$, so that the initial average distance between driven particles is many times larger than the pair-interaction length. Thus, the attractive force between driven particles is mediated by the distortion field produced in the background particles. If the fraction 


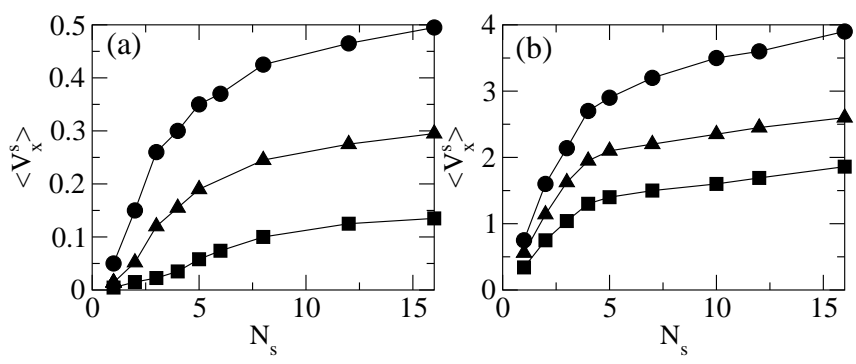

FIG. 3: $\left\langle V_{x}^{s}\right\rangle$ vs the number of particles in the string $N_{s}$. (a) The same system as in Fig. 1(a) with $q_{D} / q=6.67$ for $F_{D}=3.0$ (squares), 4.0 (triangles), and 5.0 (circles). (b) The same system as in (a) for $q_{D} / q=1.33$ and $F_{D}=0.5$ (squares), 0.75 (triangles), and 1.0 (circles).

is increased as much as an order of magnitude up to $N_{D} / N_{c}=0.1$, the preferred length of $\left\langle N_{s}\right\rangle=5$ to 6 persists. We note that if $N_{D} / N_{c}$ is too high, finite size effects due to the boundary conditions become important.

In order to illustrate the intermittent behavior and the velocity increase as the number of colloids in a string increases, in Fig. 2(a) we plot a typical time series of the velocity in the direction of drive $V_{x}$ for one of the driven colloids from the system in Fig. 1(a). Here $V_{x}(t)$ shows a series of well defined increasing or decreasing jumps, with a roughly constant velocity between the jumps. The lower value that $V_{x}$ takes is around 0.39 corresponding to the average velocity of a driven particle that is not in a string. There are several places where $V_{x}=1.45$ which corresponds to the average velocity of a string with $N_{s}=$ 5. Additionally, there are some plateaus near a value of $V_{x}=0.7$ which corresponds to $N_{s}=3$. Fig. 2(a) also shows that for short times, $V_{x}>1.5$, which corresponds to strings with $N_{s}>6$. This figure shows that the driven colloid is exiting and joining strings of different lengths.

Fig. 2(a) is only a portion of a much larger time series which covers $10^{7} \mathrm{MD}$ steps. In Fig. 2(c) we plot the histogram of this entire time series along with the histogram of $V_{x}$ (dotted line) for a case where there is only one driven particle in the system so that strings do not form. The $N_{D}=1$ curve shows a single peak in $P\left(V_{x}\right)$ near $V_{x}=0.35$, which falls near but slightly below the first peak in $P\left(V_{x}\right)$ for the multiple driven particle system. Thus, the lowest value of $V_{x}$ in Fig. 2(a) corresponds to time periods when the driven colloid is moving individually and is not part of a string. Additionally, there is a small peak in $P\left(V_{x}\right)$ for the $N_{D}=20$ system near $V_{x}=0.8$, which corresponds to the formation of strings with $N_{s}=2$. There is a large peak in $P\left(V_{x}\right)$ around $V_{x}=1.45$ corresponding to $N_{s}=5$ and 6 . For $V_{x}>1.5$ there is a rapid decrease in $P\left(V_{x}\right)$. This result shows that there is a preferred string length of $\left\langle N_{s}\right\rangle \approx 5$ for the $N_{D}=20$ system. The average velocity of a driven particle in the $N_{D}=20$ case is much larger than the average velocity in the $N_{D}=1$ case, even through the fraction of driven particles is only $N_{D} / N_{c}=0.01$.
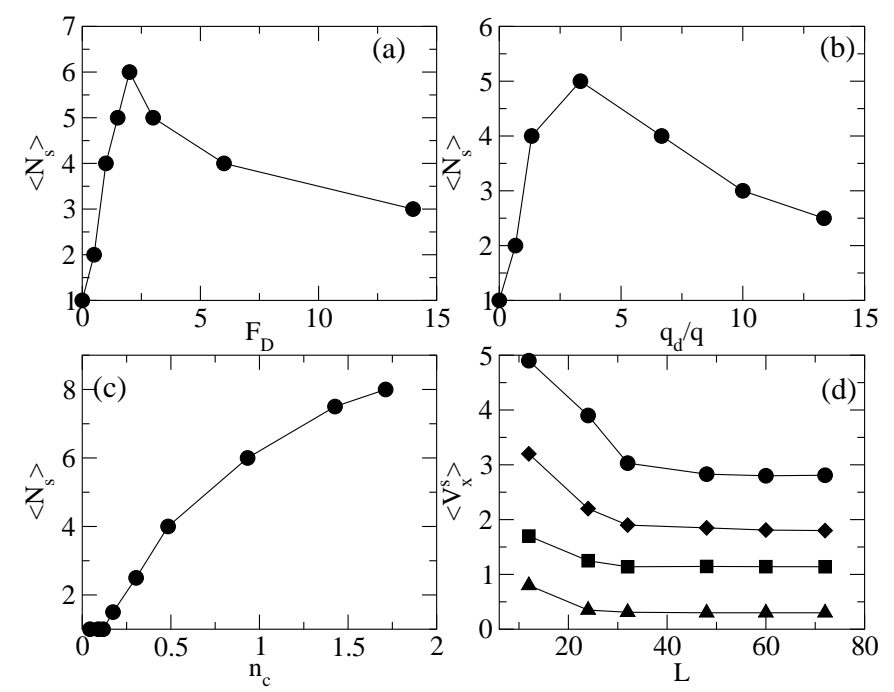

FIG. 4: (a) The most probable number of particles in a string $\left\langle N_{s}\right\rangle$ vs $F_{D}$ for the system in Fig 1. (b) $\left\langle N_{s}\right\rangle$ vs $q_{D}$ for the same system as in (a) for $F_{D}=1.0$. (c) $\left\langle N_{s}\right\rangle$ vs $n_{c}$ for $q_{D}=6.67$ and $F_{D}=2.0$. (d) $\left\langle V_{x}^{s}\right\rangle$ vs system size $L$ for the system in Fig. 1 for $F_{D}=1.5$ (triangles), 3.0 (squares), 4.5 (diamonds), and 6.0 (circles).

Driven particles traveling in strings show a more pronounced transverse wandering motion than individual driven particles. In Fig. 2(b), we plot the time series for the velocity transverse to the driving force, $V_{y}$, for the same particle as in Fig. 2(a). Here $\left\langle V_{y}\right\rangle=0$, and there are numerous spikelike events in the positive and negative directions. In Fig. 2(d) we plot the histogram of the entire time series of $V_{y}$ (solid line) along with the same histogram for a system containing only a single driven particle (dashed line). The $N_{D}=1$ curve shows a well defined Gaussian distribution of $P\left(V_{y}\right)$ centered at zero. For $N_{D}=20$, the spread in $V_{y}$ is much larger and the fluctuations are non-Gaussian, as indicated by the presence of large tails in the distribution. There are an excess of events at larger values of $\left|V_{y}\right|$ which result from the fact that the particles in a chain move in a correlated fashion. The non-Gaussian statistics also indicate that the strings have transverse superdiffusive behavior.

We next examine the average velocity of the strings $\left\langle V_{x}^{s}\right\rangle$ vs $N_{s}$, which we plot in Fig. 3(a) for systems with the same parameters as in Fig. 1 at $F_{D}=3.0,4.0$, and 5.0. The bottom curve, which corresponds directly to the system in Figs. 1 and 2, shows that for $N_{s}=1.0$, $\left\langle V_{x}^{s}\right\rangle=0.35$, while for $N_{s}=5$ and $6,\left\langle V_{x}^{s}\right\rangle \approx 0.15$. The string velocity increases rapidly with $N_{s}$ for $N_{s}<5$ and then increases much more slowly with $N_{s}$. The longer strings become considerably more winding and experience additional frictional drag, whereas the shorter strings move rigidly. Due to the meandering of the longer strings, there is a tendency for the driven particles at the back of the string to be ripped away from the string very quickly. For curves with higher $F_{D}$, there is still a monotonic increase in the average velocity with $N_{s}$. In order to 
show that this phenomenon is robust over a wide range of parameters, in Fig. 3(b) we plot $\left\langle V_{x}^{s}\right\rangle$ vs $N_{s}$ for a system with $q_{D} / q=1.33$ for $F_{D}=0.5,0.75$, and 1.0. For these parameters we again observe the string formation and an increase in $\left\langle V_{x}^{s}\right\rangle$ with $N_{s}$. The best functional fit we find is $\left\langle V_{x}^{s}\right\rangle \propto \ln \left(N_{s}\right)$; however, other forms can also be fit, such as two linear fits for the system with $q_{D}=1.33$.

In Fig. 4(a) we show the most probable number of particles $\left\langle N_{s}\right\rangle$ in a string for the same system in Fig. 1 for varied $F_{D}$. Here $\left\langle N_{s}\right\rangle$ goes through a maximum as a function of $F_{D}$. As $F_{D}$ decreases below $F_{D}<0.2$, the distortion created by the driven particles in the surrounding background particles decreases, and therefore there is less attraction between the driven particles. At high $F_{D}$, the velocity fluctuations along the string become important, which tends to limit the string length. In Fig. 4(b) we show that $\left\langle N_{s}\right\rangle$ for a system with a fixed $F_{D}=1.0$ also goes through a maximum as a function of $q_{D} / q$. For small $q_{D} / q$, the driven particles produce very little distortion in the surrounding medium so there is little attraction between them. For large $q_{D / q}$, the repulsion between the driven particles becomes more prominent and the average velocity drops, which again reduces the distortion field. In Fig. 4(c) we plot $\left\langle N_{s}\right\rangle$ vs the particle density $n_{c}$ for a system with $q_{D}=6.67$ and $F_{D}=2.0$. At low $n_{c}$, all the particles are far apart and the distortion in the bath particles is reduced or absent. As $n_{c}$ increases, the particle-particle interactions are enhanced and the string can grow longer. Presumably for very high $n_{c},\left\langle N_{s}\right\rangle$ will decrease again as the average velocity will have to be reduced. We are not able to access particle densities in this range.

In Fig. $4(\mathrm{~d})$ we plot $\left\langle V_{x}^{s}\right\rangle$ vs the system size $L$ for $F_{D}=1.5$ (lower curve), 3, 4.5, and 6.0 (upper curve). In each case, $\left\langle V_{x}^{s}\right\rangle$ initially decreases with $L$ but reaches a saturation value by $L \approx 40$. The change in $\left\langle V_{x}^{s}\right\rangle$ at small $L$ occurs because, in small systems, the periodic boundary conditions allow driven colloids to interact strongly with their own distortion trails. For larger $F_{D}$, larger system sizes must be used to avoid this effect. In addition to the increase in $\left\langle V_{x}^{s}\right\rangle$ in the small systems, $\left\langle N_{s}\right\rangle$ also increases for the smallest systems. This emphasizes the fact that sufficiently large systems must be considered in order to avoid finite size effects. We have used $L=48$ throughout this work, which is large enough to avoid finite size effects for the quantities we have measured.

The attraction between the driven particles is mediated by the distortion in the surrounding bath of colloids. The concept of nonequilibrium depletion forces, which are highly anisotropic and have both an attractive and a repulsive portion, was introduced recently for a case where two large colloidal particles have an additional bath of smaller particles flowing past them [10]. The surrounding particle density is higher in front of the large particle and lower in back, which causes an anisotropic attraction force between the large colloids that aligns them with the direction of flow. Although this model was only studied for two particles, it seems to capture several of the features that we find in our simulations, including the alignment of the strings in the direction of drive. This suggests that nonequilibrium depletion force models could be applied for multiple large particles. The longer strings move faster since the front particle has additional particles pushing on it from the back. Since the chains are aligned, the cross section with the background particles remains the same as for a single driven particle; however, the string is coupled $N_{s}$ times more strongly to the external field. As longer strings become increasingly meandering, the cross section with the background particles increases and limits the speed that long strings can attain.

In conclusion, we have investigated a system of repulsively interacting Yukawa particles which form a disordered background for a small fraction of Yukawa particles that couple to an external drive. In this system, despite the fact that all of the pair-wise interactions are repulsive, there is an emergent effective attraction between the driven particles which results in the formation of onedimensional intermittent stringlike structures. The velocity of a string increases with the number of particles in the string. The attraction between the driven particles is mediated by the distortion of the surrounding particles and is likely a result of the recently proposed nonequilibrium depletion forces. Longer strings move faster than shorter strings due to the additional pushing force from the back particles in the string. This system represents a new class of collective transport where multiple interacting colloids are driven through a deformable disordered background, which is distinct from the case where the background disorder is quenched.

We thank E. Ben-Naim for useful discussions. This work was supported by the U.S. DoE under Contract No. W-7405-ENG-36.

[1] S. Bhattacharya and M.J. Higgins, Phys. Rev. Lett. 70, 2617 (1993); A.E. Koshelev and V.M. Vinokur, ibid. 73, 3580 (1994); G. Li et al., ibid. 96, 017009 (2006).

[2] G. Grüner, Rev. Mod. Phys. 60, 1129 (1988).

[3] J. Tekic, O.M. Braun, and B. Hu, Phys. Rev. E 71, 026104 (2005).

[4] P. Habdas, D. Schaar, A.C. Levitt, and E.R. Weeks, Europhys. Lett. 67, 477 (2004); and to be published.

[5] M.B. Hastings, C.J. Olson Reichhardt, and C. Reichhardt, Phys. Rev. Lett. 90, 098302 (2003).

[6] B. Schmittmann, K. Hwang, and R.K.P. Zia, Europhys. Lett. 19, 19 (1992); D. Helbing, I.J. Farkas, and T. Vicsek, Phys. Rev. Lett. 84, 1240 (2000).

[7] J. Dzubiella, G.P. Hoffmann and H. Löwen, Phys. Rev. E 65, 21402 (2002); J. Chakrabarti, J. Dzubiella, and H. Löwen, ibid. 70, 012401 (2004).

[8] D. Helbing, Rev. Mod. Phys. 73, 1067 (2001).

[9] M.E. Leunissen et al., Nature (London) 437, 235 (2005).

[10] J. Dzubiella, H. Löwen, and C.N. Likos, Phys. Rev. Lett. 91, 248301 (2003). 